\title{
Russian Federation State Prize in Science and Technology for 2009
}

\begin{abstract}
The Russian Federation State Prize in Science and Technology for 2009 honoring scientists for "A Set of Scientific Works on the Development of Laser and Information Technologies in Medicine" (Presidential Decree no. 678, June 6, 2010) was awarded to V.Ya. Panchenko, doctor of physical and mathematical sciences, academician of the Russian Academy of Sciences, director of the Institute of Laser and Information Technologies, Russian Academy of Sciences; to A.A. Potapov, doctor of medical sciences, Russian Academy of Medical Sciences, deputy director of the Burdenko Neurosurgery Research Institute, Medical Academy of Medical Sciences; and to V.I. Chissov, doctor of medical sciences, academician of the Russian Academy of Medical Sciences, director of Herzen's Cancer Research Institute, Moscow.
\end{abstract}

In the work of these scientists, a system for the remote production of individual implants and biomodels based on tomography data of the evaluation of pre-surgery patients via the Internet to a center for rapid prototyping and manufacturing was established for the first time ever. Original locally conceived systems for rapid prototyping, namely, laser systems and selective laser sintering of micro-, nano-, and biocompatible materials, were designed and manufactured. Biomodels created based on these materials are being used for pre-surgery planning.

This technology of preoperative biomodeling is being widely used in neurosurgery, oncology, and reconstructive surgery at many hospitals in different regions of Russia and other countries. The effectiveness of operational preplanning using biomodels is demonstrated in the significant reduction in time and in the improvement of treatment outcomes in neurosurgery, maxillofacial surgery, reconstructive surgery, and oncology.

A huge amount of problems related to traumatic brain injury are solved through neurosurgery. For the first time, technology and methods for obtaining and producing stereo lithographic models that are fully congruent to real facial and cranial defects are being developed, therefore radically changing plastic and reconstructive microsurgery and allowing for the accumulation of the largest body of clinical material both in the country and in the world.

Technology to manufacture individual implants from various biocompatible materials and molds for its pro- duction using the "symmetric stereo lithography" method, as well as a database of "online donors," are being developed.

For the first

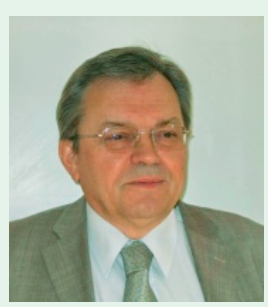

V.Ya. Panchenko

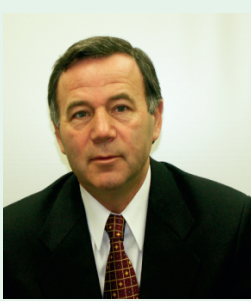

A. A. Potapov

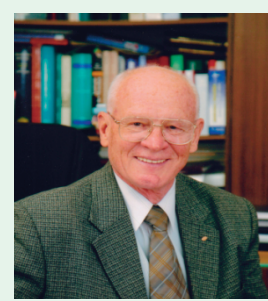

V.I. Chissov time, a special form of traumatic brain injury (crush syndrome of the head) has been described. Several other technologies are being developed and implemented in practice, such as computer simulation of preoperative craniocerebral operations and intraoperative navigation technology, including three-dimensional CT, MRI, and ultrasound images; optical fluorescence; neurophysiologic mapping; and neuromonitoring.

These concepts and technology for reconstructive neurosurgery, which yield restoration of the structural integrity of the brain's functionality and the individual configuration of the skull, as well as the soft covers of the head based on modern laser information technologies, are a priority.

A novel approach is being pursued in the field of cancer surgery in which a complex set of laser technologies, such as spiral CT, MRI, and 3D ultrasound, constitute the foundation for the surgical planning of anatomically complex locations of tumors; the data obtained combine laser information technologies, creating a real three-dimensional model of the diseased organ. This proves that it is possible to successfully surgically treat a tumor that affects the skull's base, cranio-maxillo-facial region, spine, larynx, and trachea. Preopera- tive planning for the real prototype, obtained by laser stereo lithographic technology, allows to preserve vital organs and improve the radical removal of tumors. For the first time ever mutual dependence between cancer and plastic treatment components has been established; radical tumor removal leads to defects incompatible with life and requires immediate and safe reconstruction, which is impossible without an accurate evaluation of the level and composition of the plastic material with location on real biomodels.

A new generation of intelligent laser surgical systems have been designed and built. The laser installation in real time defines the boundaries of the treated area. Testing of this new installation revealed a new possibility for the use of the surgical laser in different modes, depending on various conditions. The system opens entirely new opportunities for low-traumatic and organsaving operations.

Laser information technologies are being introduced in other medical fields, such as ophthalmology, cardiac surgery, etc., through the creation of specialized tasks geared to these complexes.

The next issue will contain a detailed publication from the Russian State Prize winners. 